\title{
Difficulties in diagnosing multisystem Langerhans cell histiocytosis in children: a case report
}

\author{
Michalina Jezierska1', Joanna Stefanowicz'1, Anna Płoszyńska' ', Agnieszka Szlagatys-Sidorkiewicz², \\ Mirosława Dubaniewicz-Wybieralska³, Elżbieta Adamkiewicz-Drożyńska' \\ 'Department of Paediatrics, Haematology, and Oncology, Medical University of Gdansk, Gdansk, Poland \\ 2Department of Paediatrics, Gastroenterology, Allergology and Paediatrics Nutrition, Medical University of Gdansk, \\ Gdansk, Poland \\ ${ }^{3}$ Department of Radiology, Medical University of Gdansk, Gdansk, Poland
}

\section{ABSTRACT}

Langerhans cell histiocytosis (LCH) is a rare disease with an unclear aetiology and extremely heterogenous clinical symptomatology. In the following manuscript, we discuss a case of a 3.5-year-old boy diagnosed with the multisystem form of LCH (MS-LCH) with involvement of the digestive tract, liver, bones, and skin. An increased abdominal circumference and diarrhoea were the first symptoms of the disease presented by the patient. The final diagnosis was made approximately 12-18 months after the early manifestations of the disease were reported. This case study clearly shows that in spite of significant progress in the health sciences, LCH still poses serious diagnostic difficulties, which may result in a delay in treatment and thus negatively affect the patient's prognosis.

KEY WORDS:

children, Langerhans cell histiocytosis, hepatomegaly, chronic diarrhoea.

\section{INTRODUCTION}

Langerhans cell histiocytosis $(\mathrm{LCH})$ is a rare disease. There is still some debate as to whether it should be classified as a neoplasia or as an immunological process. Considerable telomere shortening and clonal proliferation of Langerhans cells are found in this disease, and approximately $75 \%$ of patients with $\mathrm{LCH}$ express the V600E mutation of the BRAF gene or MAP2K1 mutation, which may help to classify LCH as the neoplasm [1]. However, there are also arguments supporting the second possibility, such as increased expression of cytokines within the focus areas of the disease, especially interleukin-17 (IL-17), and the presence of immature Langerhans cells in the infection foci [1].
The current classification of the disease is based on clinical criteria. More frequently, in $65 \%$ of cases, a single-system form of histiocytosis (SS-LCH) with involvement of one organ or system is observed. A multisystem form of histiocytosis (MS-LCH) with the involvement of at least two organs or systems occurs less frequently and has a worse prognosis [2-5].

As well as there being completely asymptomatic forms of $\mathrm{LCH}$, there are also forms with non-characteristic symptoms, such as a deterioration of the general condition, fever, and loss of appetite or body mass, as well as forms with symptoms resulting from the involvement of particular organs or systems, such as the bones (80\%), skin (33\%), pituitary gland (25\%), liver (15\%), spleen (15\%), haematopoietic system (15\%), lungs (15\%), lymph

\section{ADDRESS FOR CORRESPONDENCE:}

Joanna Stefanowicz, Department of Paediatrics, Haematology, and Oncology, Medical University of Gdansk,

7 Dębinki St., 80-211 Gdansk, Poland, ORCID: 0000-0001-8014-4263, e-mail: jstefanowicz@gumed.edu.pl 


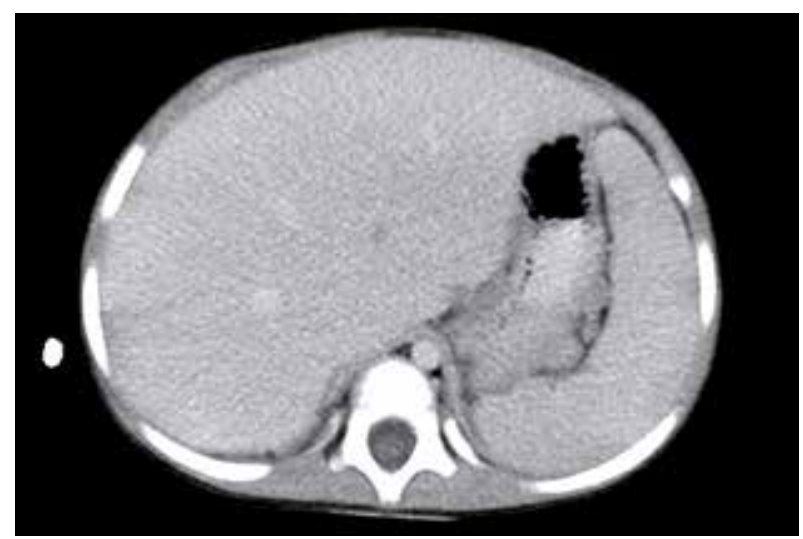

FIGURE 1. Computed tomography of the abdomen - a very large liver [14]

nodes (5-10\%), central nervous system apart from the pituitary gland (2-4\%), thymus, and digestive tract [4].

\section{CASE REPORT}

A 3.5-year-old boy was admitted to the oncology department in January 2012.

For approximately 12-18 months prior to hospitalisation, the child was observed because he had an increased abdominal circumference and diarrhoea. For approximately 12 months, he followed a low-lactose diet with little improvement in symptoms. In November 2011 , the child had febrile states $\left(38.5^{\circ} \mathrm{C}\right)$ and a decreased appetite. In December 2011, the child was hospitalised to undergo more thorough diagnostic tests. At this time, the condition of the patient was considered to be quite good. A physical examination revealed deficits of body mass and height (third centile), as well as paleness, scalp lesions defined as crusta lactea, a significant increase in the abdominal circumference, and an enlarged liver $(+3 \mathrm{~cm})$. The boy had significant microcytic anaemia (Hb: $7.7 \mathrm{~g} / \mathrm{dl}, \mathrm{MCV}$ : $70 \mathrm{fl}$ ). Laboratory tests revealed that the child had low levels of iron $(37 \mu \mathrm{g} / \mathrm{dl})$ and ferritin $(27.3 \mathrm{ng} / \mathrm{ml})$, increased levels of inflammation parameters (CRP: $28.2 \mathrm{mg} / \mathrm{l}$, PCT: $2.2 \mathrm{ng} / \mathrm{ml}$ ), slight hypoproteinaemia (TP: $61 \mathrm{~g} / \mathrm{l}$ ), and hypoalbuminaemia (32 $\mathrm{g} / \mathrm{l})$. USG of the abdomen revealed the presence of hepatomegaly and multiple enlarged mesenteric lymph nodes. Finally, the child was diagnosed with parvovirus B19 infection. Additionally, the presence of Clostridium difficile toxins in the stool was reported. The patient was administered metronidazole, probiotics, and iron supplementation. Initially, an improvement of his general state and a normalisation of his body temperature and inflammation parameters were observed. Soon thereafter, the patient developed a recurrence of fever, and laboratory tests revealed that he had an increased level of CRP. It was radiologically documented that the patient had pneumonia. Due to the infection, a scheduled gastroenterological diagnostic evaluation (including gastroscopy) was cancelled, and when clinical improvement of the patient was observed, the patient was discharged from the hospital.

Eighteen days later, the boy had to be hospitalised again due to a fever and increasing signs of an inflammatory condition without noticeable signs of infection by physical examination and microbiological tests. He was treated empirically with cefotaxime and metronidazole but showed no improvement. Soon, the patient developed pain in the abdomen and vomiting, and on the eighth day the boy was finally transferred to a surgical ward under suspicion of having acute abdomen. Following a 10-hour observation the antibiotic therapy was modified (meropenem was used) and red cell concentrate ( $\mathrm{Hb}: 7.1 \mathrm{~g} / \mathrm{dl}$ ) was transfused, the boy was transferred back to the paediatric ward. The most alarming signs were the boy's large, bloated abdomen and significant hepatomegaly (with the liver reaching the ileum). There was a suspicion of $\mathrm{LCH}$ due to the patient presenting scalp lesions but the performed X-ray of the skull was correct. Because the patient had a persistent fever and his inflammation parameters increased further (CRP: $112.1 \mathrm{mg} / \mathrm{l}$ ), the antibiotic therapy was modified again (metronidazole was replaced with vancomycin), and biopsies of the liver and bone marrow were performed. In the follow-up X-ray of the thorax, foci of the rarefaction of the bone structure were found in the left scapula, left humerus and eighth right rib. A computed tomography (CT) scan revealed a single osteolytic focus in the L1 shaft, aside from the hepatomegaly (Fig. 1) and bone lesions mentioned above. $\mathrm{LCH}$ was again suspected, and an additional biopsy of the scalp was performed.

Histopathological examination of the skin and liver tissues confirmed the LCH diagnosis (IHC: LCA+, S100+, $\mathrm{CD} 1 \mathrm{a}+$, tryptase + ). The bone marrow biopsy produced normal results.

The boy started chemotherapy in accordance with the Polish Paediatric Leukaemia/Lymphoma Study Group Treatment Program (etoposide, vinblastine, and prednisone). Initially, an improvement of the patient's general condition was reported; the fever and skin lesions subsided and the liver decreased in size. However, laboratory tests still indicated hypoalbuminaemia. After the administration of five cycles of chemotherapy, which is when steroids were gradually discontinued in accordance with the protocol, the patient suffered from a recurrent fever that was resistant to the administered antibiotic therapy, and he developed a mild proptosis of his left eye. There were no abnormalities in the neurological and ophthalmological examinations. Magnetic resonance imaging (MRI) of the brain, facial bones, and orbital cavities was performed and revealed the presence of a semilunar tissue structure surrounding the optic nerve (Fig. 2). The patient did not manifest symptoms of diabetes insipidus and other endocrinopathies. Therefore, endocrine tests were not performed. A progression of the disease was reported. It was decided that a change in the chemotherapy 
would be made in accordance with the LCH-S-2005 program and, due to suspicion of steroid addiction, that the steroids would be continued.

The boy received five cycles of chemotherapy with cladribine and cytoside arabinoside, followed by a threestage maintenance chemotherapy (cladribine, vinblastine, 6-mercaptopurine, and methotrexate). The steroid therapy was reincorporated due to lesions in the left orbital cavity still being visible in the scan.

The chemotherapy was complicated by considerable myelosuppression and a catheter-related blood infection. The patient required prolonged regeneration intervals, G-CSF administration, transfusion of blood components, and antibiotic therapy. Removal the catheter with reimplantation of the vascular access port was also necessary. During the oncological treatment, he had hypertension, which required pharmacotherapy.

The boy is currently in the remission state of the disease.

\section{DISCUSSION}

Our patient, whose clinical manifestations of the disease included fever, an increase of the abdominal circumference with hepatomegaly, chronic diarrhoea, physical growth inhibition, and skin lesions, was finally diagnosed with MS-LCH.

For this patient, intensive diagnostics took approximately 1.5 months, but according to his parents, the first symptoms of the disease, such as enlargement of the abdomen and diarrhoea, appeared 12-18 months earlier. These signs gave rise to the suspicion of food intolerance, which resulted in the patient starting a diet. At this stage, the initial diagnosis seemed to be conclusive, and similar sequences of events often accompany the descriptions of patients diagnosed with $\mathrm{LCH}$ with digestive tract involvement [6]. This organ localisation of the disease is very rare $(<1 \%)$ and usually affects children under one year of age. The most typical clinical manifestations are: presence of blood in the stool, diarrhoea, constipation, absorption disorders, and enteropathy, including protein loss $[6,7]$. Pain of the abdomen and vomiting occur less often. However, these symptoms were the reason that our patient was hospitalised in the surgical ward under suspicion of acute abdomen. Perforation of the intestine is very rare [6]. Elimination diets may initially result in clinical improvement; however, in the long run, they will not cause the ailments to subside. Furthermore, the patient presented with fever without noticeable signs of infection and a decrease in appetite. He was diagnosed as being underweight and as having height deficiency, as well as skin lesions, hepatomegaly, microcytic anaemia, increased inflammation parameters, hypoproteinaemia, and hypoalbuminaemia. It is noteworthy that a scheduled gastroscopy had to be postponed due to the deterioration of the boy's general condition. The authors of previous studies on $\mathrm{LCH}$ patients with digestive tract involvement

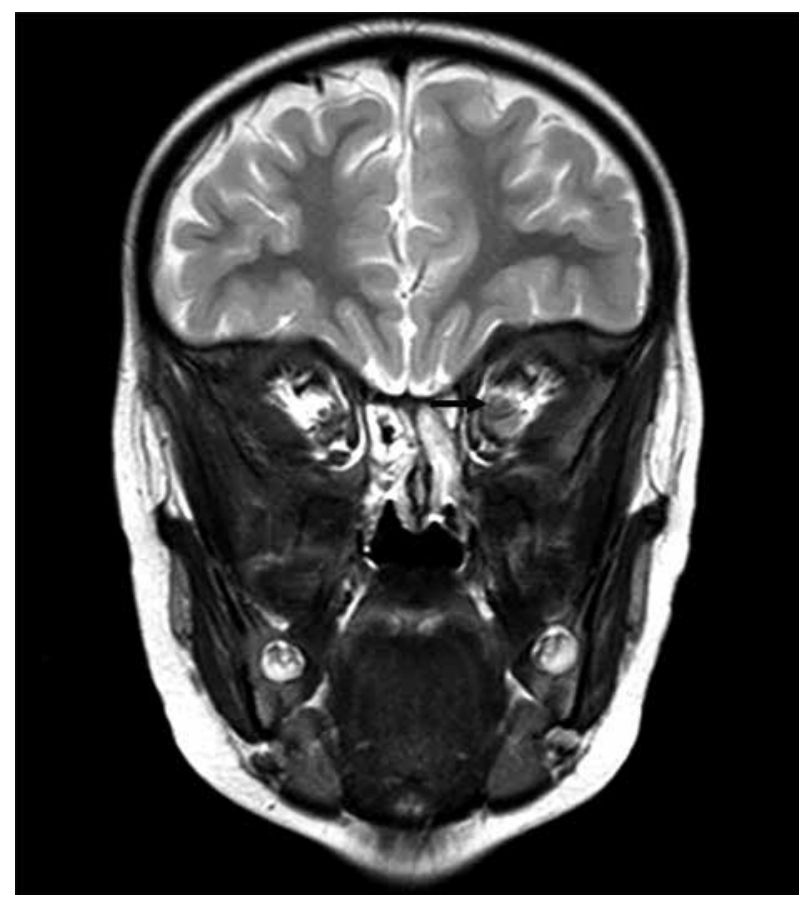

FIGURE 2. Magnetic resonance imaging of the orbits - a semilunar tissue structure surrounding the optic nerve

agree that for patients reporting gastrointestinal symptoms, especially for those with accompanying skin lesions and whose elimination diet proves to be ineffective, an endoscopic biopsy of the digestive tract as soon as possible should be performed [7].

Skin lesions observed in the course of $\mathrm{LCH}$ are non-characteristic. According to medical publications, the average time from the moment when the first skin lesions appear to the moment when the diagnosis is established is three months [8]. The clinical manifestation includes follicles and vesicae in early childhood years. In slightly older children, follicular-erythematous and a scaling rash may appear, mainly located on the scalp and behind the ears, as in the case of our patient, or in the napkin or armpit area. Nodules or lesions of haemorrhagic nature are less common $[2,4]$. It is not clear how isolated skin lesions progress to MS-LCH. The medical literature estimates the risk this disease course is between $0 \%$ and $60 \%[8,9]$.

Another organ that demonstrated localisation of LCH in our patient was the liver. The liver may be involved as a result of a primary process, as well as a secondary process, namely, infiltrations of the lymph nodes in the hepatic hilum or hypertrophy of Kupffer cells and hyperplasia secondary to the generic inflammatory response $[10,11]$. Our patient presented with the most common sign of involvement of the liver: hepatomegaly, which was accompanied by the organ's dysfunction (hypoproteinaemia and hypoalbuminaemia). Other forms of liver dysfunctions in LCH are: hyperbilirubinaemia, hypertransaminasaemia and coagulation disorders [5, 11, 12]. To diagnose liver involvement in $\mathrm{LCH}$, it is sufficient to observe a liver enlargement $>3 \mathrm{~cm}$ below the costal mar- 
gin in the midclavicular line in USG [3]. Biopsy of the organ is not necessary if the diagnosis is established on the basis of a histopathological examination of specimens collected from different locations. According to medical publications, if hepatomegaly is accompanied by sclerosing cholangitis or cirrhosis, the biopsy may even be non-diagnostic [4].

The last symptom, which completed the picture of the disease in our patient, was the presence of lytic foci in the bones, which is the most common clinical manifestation of LCH [13]. Lesions are usually located in the flat bones, vertebral column, and long bones. Some lesions are very characteristic of $\mathrm{LCH}$, e.g. lesions located in the cranial bones $[10,13,14]$, and our patient therefore received an $\mathrm{X}$-ray examination of the cranium when he was first suspected of having histiocytosis. Bone localisation of $\mathrm{LCH}$ is also connected with one-sided involvement of the orbital cavity, which may be accompanied by histiocytic infiltrations [15] in the form of tissue masses located in the orbital cavity.

The anaemia he presented with was probably secondary to iron deficiency in the course of malabsorption and met neither the criteria for anaemia-accompanying chronic diseases nor the criteria for anaemia resulting from primary bone marrow involvement.

After the retrospective analysis, it seems that the presented patient, due to the multi-systemic form of the disease, should also undergo a thorough endocrine evaluation, which was omitted due to the lack of clinical symptoms of endocrinopathy. The prognosis in this case was not good. The boy presented with two of the worst prognostic factors, i.e. involvement of "risk organs" and an inadequate response to induction chemotherapy. The intensification of chemotherapy allowed for final remission.

What may arouse controversies is the number of invasive procedures performed before establishing the final diagnosis. Undoubtedly, the decisive factor was a very rare, non-characteristic, clinical symptomatology.

\section{CONCLUSIONS}

This study emphasises that in the case of recurrent fever without noticeable infection, resistant to the administered antibiotic, intensive diagnostics to exclude rare diseases should be implemented. In the case of atypical course of the disease imitating infection associated with the presence of lytic foci in the bones, we should remember about $\mathrm{LCH}$.

In summary, this study is further evidence exhibiting how serious the diagnostic dilemmas are that every practising paediatrician may face and the difficult decisions they may have to make.

\section{DISCLOSURE}

The authors declare no conflict of interest.

\section{REFERENCES}

1. Grana N. Langerhans cell histiocytosis. Cancer Control 2014; 21: 328-334.

2. Morren MA, Vanden Broecke K, Vangeebergen E, et al. Diverse cutaneous presentations of Langerhans cell histiocytosis in children: A retrospective cohort study. Pediatr Blood Cancer 2016; 63: 486-492.

3. Langerhans cell histiocytosis. Histiocyte Society Evaluation and Treatment Guidelines. April 2009.

4. Haupt R, Minkov M, Astigarraga I, et al. Langerhans cell histiocytosis (LCH): Guidelines for diagnosis, clinical work-up, and treatment for patient till age of 18 years. Pediatr Blood Cancer 2013; 60: 175-184.

5. Wong A, Ortiz-Neira CL, Reslan WA, et al. Liver involvement in Langerhans cell histiocytosis. Pediatr Radiolog 2006; 36: 1105-1107.

6. Hait E, Liang M, Degar B, et al. Gastrointestinal tract involvement in Langerhans cell histiocytosis: Case report and literature review. Pediatrics 2006; 118: 1593-1599.

7. Shima H, Takahashi T, Shimada H. Protein-losing enteropathy caused by gastroitestinal tract-involved Langerhans cell histiocytosis. Pediatrics 2010; 25: 426-432.

8. Simko SJ, Garmezy B, Abhyankar H, et al. Differentiating skin-limited and multisystem Langerhans cell histiocytosis. Pediatr Blood Cancer 2014; 65: 990-996.

9. Ng SS-Y, Koh MJ-A, Tay Y-K. Cutaneous Langerhans cell histiocytosis: Study of Asian children show good overall prognosis. Acta Paediatr 2013; 102: 514-518.

10. Egeler R. LCH: Symptoms, Diagnosis and Treatment. https://histiocytesociety.org/document.doc?id=49 (access: 30 July 2018).

11. Yi X, Han T, Zai H, et al. Liver involvement of Langerhans cell histiocytosis in children. Int J Clin Exp Med 2015; 8: 7089-7106.

12. Guthery SL, Heubi JE. Liver involvement in childhood histiocytic syndromes. Curr Opin Gastroenterol 2001; 17: 474-478.

13. Lau LM, Stuurman K, Weitzman S. Skeletal Langerhans cell histiocytosis in children: Permanent consequences and health-related quality of life in long-term survivors. Pediatr Blood Cancer 2008; 50: 607-612.

14. Jezierska M, Stefanowicz J, Romanowicz G, et al. Langerhans cell histiocytosis in children - a disease with many faces. Recent advances in pathogenesis, diagnostic examinations and treatment. Adv Dermatol Allergol 2018; 1: 6-17.

15. Herwing MC, Wojno T, Zhang Q, et al. Langerhans cell histiocytosis of the orbit: five clinicopathologic cases and review of the literature. Surv Ophthalmol 2013; 58: 330-339. 